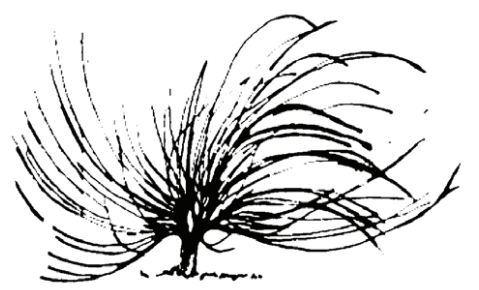

\title{
Una Aproximación Sociopolítica al Desabastecimiento, Tarifas, Subsidios y Tandeos Relativos a los Servicios Hídricos
}

Cruz García Lirios ${ }^{1}$

Universidad Nacional

Autónoma de México

México

garcialirios@yahoo.com

José Marcos Bustos Aguayo

Universidad Nacional

Autónoma de México

México

marcos.bustos@unam.mx

José Alfonso Aguilar Fuentes 5

Universidad Autónoma del

Estado de México

México

jaaguilarf@uamex.mx
Javier Carreón Guillén ${ }^{2}$

Universidad Nacional

Autónoma de México

México

javierg@unam.mx

Jorge Hernández Valdés ${ }^{4}$

Universidad Nacional

Autónoma de México

México

jorheval@unam.mx

Francisco Javier Rosas Ferrusca ${ }^{6}$

Universidad Autónoma del

Estado de México

México

fjrosasf@uaemex.mx

\section{(C) (1) $\Theta \Theta$}

Recibido: 6 de agosto de 2014-Aprobado: 13 de abril de 2015

1 Estudios de Doctorado en Psicología Social y Ambiental, UNAM. Profesor de asignatura, UAEMEX-UAP Huehuetoca.

2 Doctor en Administración, UNAM. Profesor titular "C", ENTS. Adscrito al SNI, nivel I.

3 Doctor en Psicología Social y Ambiental, UNAM. Profesor Titular "A", FES Zaragoza. Adscrito al SNI, nivel I.

4 Estudios de Doctorado en Ciencia Política, UNAM. Profesor Titular “A”, ENTS.

5 Doctor en Educación UAM-Xochimilco. Profesor de Tiempo Completo UAEMEX-UAP Huehuetoca.

6 Doctor en Administración Pública, INAP. Profesor de Tiempo Completo, UAEMEX-UAP Huehuetoca. 


\title{
Resumen
}

El presente trabajo revisa documentación institucional, científica y tecnológica relativa a la disponibilidad de agua, consumo humano, política de tandeo, establecimiento de tarifas, subvenciones del precio unitario del agua y estilos de vida antropocéntricos y ecoperiféricos con la finalidad de comparar el enfoque psicosocial con la aproximación económica. Para tal propósito, se buscó información en bases de datos indexadas con registro ISSN y DOI en DIALNET, LATINDEX y REDALYC durante el periodo que va de enero 2010 a octubre 2014 . El presente escrito, en referencia al estado del conocimiento, revela que ambos enfoques han sido rebasados por las relaciones sociopolíticas en coyunturas pre y post electorales. Por consiguiente, se propone el abordaje de las problemáticas desde una concepción sociopolítica que explique y anticipe las confrontaciones entre los actores en contextos de politización del abastecimiento de agua.

Palabras clave: recursos hídricos, disponibilidad, tarifas, subsidios, tandeo, comicios

\begin{abstract}
This paper reviews the institutional, scientific and technical documentation regarding the availability of water for human consumption, policy tandem, setting tariffs, subsidies unit price of water and anthropocentric and ecoperipheral life styles in order to compare the psychosocial approach with the economic approach. For this purpose, information was sought in databases indexed with ISSN and DOI registration DIALNET LATINDEX and REDALYC during the period from January 2010 to October 2014. This article, with regard to to the state of knowledge, reveals that both approaches have been overwhelmed by the socio-political relations in pre and post-electoral periods. Therefore, addressing the problem is proposed from a sociopolitical concept to explain and anticipate confrontations between actors in contexts of politicization of water.
\end{abstract}

Keywords: water resource availability, rates, subsidies, tandem, elections 


\section{Introducción}

El presente trabajo incluye tres objetivos: 1) contrasta dos enfoques, uno económico y otro psicológico en torno a las relaciones de dependencia entre disponibilidad de agua y consumo humano, para 2) evidenciar las diferencias que orientarían políticas públicas, sistemas de tarifas, subsidios y tandeos indicadas por correlaciones entre variables determinantes del consumo de agua a partir de una revisión del estado del conocimiento según el cual sería posible, 3) anticiparían conflictos entre usuarios y autoridades como resultado de políticas de tandeo, incremento de tarifas y reducción de subsidios.

En el marco del Desarrollo Sustentable, la psicología se ha avocado al estudio de las relaciones entre la disponibilidad hídrica per cápita y el consumo de agua. Los Estudios Psicológicos de la Sustentabilidad (EPS) han demostrado asociaciones significativas entre la cantidad de agua abastecida y el dispendio o ahorro según el volumen per cápita.

En virtud de los hallazgos mencionados, los EPS han abierto la discusión relativa al costo del servicio público de agua potable, estimado por criterios tarifarios convencionales en los que la inclusión de factores disposicionales, situacionales, culturales, cognitivos y conductuales harían más eficiente el sistema de cobros, sanciones y subsidios.

La lógica de los EPS también incidiría en el diseño e implementación de políticas públicas que permitan financiar la Acción Pública Gubernamental (APG) ante el incremento de la problemática de escasez, desabasto e insalubridad hídricas.

A partir de diagnósticos causales y correlacionales, los EPS plantean modelos teórico-estructurales que posibiliten el aumento o disminución de la tarifa de consumo considerando las relaciones asociativas entre las variables implicadas.

La correlación bivariada estadística estimada con el parámetro " $r$ " de Pearson permite una abstracción de las relaciones concretas asociadas en una variable X y una variable $Y$. La fuerza de asociación entre una variable $\mathrm{X}$ y otra variable $\mathrm{Y}$ es conocida como correlación. Se trata de un análisis en el que se establecen las relaciones asociadas entre las variables que configuran una estructura cultural, social, comunitaria, económica, política, institucional, corporativa, educativa o familiar. Dicha estructura es evidenciada en un modelo en el que las variables y los constructos explican la influencia de la estructura en los individuos. 
El modelo se construye a partir de las relaciones asociadas entre las variables para inferir sus relaciones causales. En torno a la escasez de agua, las asociaciones exógenas y endógenas entre los valores, las creencias, las percepciones, las actitudes, las habilidades y las intenciones orientan su modelización como determinantes del dispendio o ahorro de agua.

Sin embargo, los EPS parecen orientarse al establecimiento de tarifas al plantear al agua como un recurso y a los usuarios como consumidores. En un sistema de oferta, el estado provee de los servicios públicos de abasto sin considerar la tendencia de disponibilidad per cápita. En otros casos, las autoridades administrativas determinan discrecionalmente las tarifas de consumo. Los EPS han establecido relaciones significativas entre el abasto intermitente y el uso austero de agua. No obstante, tales hallazgos están desvinculados de los sistemas tarifarios puesto que los proyectos de investigación no se han planteado la posibilidad de explorar el conformismo o inconformidad de los usuarios respecto al servicio público y la política ambiental local que llevan a cabo sus gobernantes. Precisamente, exponer los alcances y límites de los EPS respecto a las problemáticas hídricas abrirá la discusión relativa a los conflictos entre autoridades encargadas de proveer el servicio de agua potable y los usuarios que reciben un volumen inferior en comparación a otras localidades y regiones. En el marco del Desarrollo Sustentable, las problemáticas hídricas de escasez, abasto irregular e insalubridad parecen ser suficientes para inhibir el confort hídrico definido como el volumen mínimo per cápita para llevar a cabo las actividades y satisfacer las necesidades básicas que asociadas con capacidades, habilidades, competencias y conocimientos permitirán a la humanidad superar los umbrales de pobreza extrema y su inclusión en los servicios públicos será un indicador de su desarrollo local y regional.

\section{Enfoque económico y aproximación psicosocial en torno a la dispo- nibilidad y consumo humano del agua}

Desde el enfoque económico, la problemática del agua supone un desbalance entre disponibilidad y consumo que sólo podrá ser resuelto si se implementa un sistema de tarifas.

En el mundo, el $97.5 \%$ del agua es salada, $2.24 \%$ es dulce y solo $1 \%$ está disponible en ríos, lagos y acuíferos para el consumo humano. 
$113000 \mathrm{~km} 3$ de agua se precipitan anualmente (United Nations Water, 2013, p. 13-18).

La disponibilidad del recurso ha disminuido paulatinamente. En 1950, sólo el continente asiático tenía una baja disponibilidad y para el año 2025 esta escasez se extenderá a los cinco continentes. El desequilibrio entre la explotación (se estiman $4600 \mathrm{~km} 3$ ) del recurso y su recarga natural afectará su disponibilidad para el consumo $(2400 \mathrm{~km} 3$ aproximadamente) en la agricultura, la industria y las actividades domésticas (United Nations Habitad, 2010, pp. 16-29).

En el caso de México, al ser el onceavo país con más población en el mundo (101,7 millones de personas), con una densidad de 52 personas por km 2 en promedio; una población menor de 15 años (33\%), el $74 \%$ vive en zonas urbanas y su ingreso per cápita al año es de 8,790 dólares norteamericanos trabajando 40 horas a la semana, su crecimiento anual es de 2,1 millones y se espera que en el 2050 aumente $48 \%$, estimando su población en 131, 7 millones para el 2030, ha sido clasificado con un índice de disponibilidad extremadamente baja con menos de 1000 metros cúbicos por habitante al año (Instituto Nacional de Estadística Geografía e Informática, 2010, pp. 3-24).

Respecto a las zonas centro y norte del país, donde el crecimiento económico es significativo, la disponibilidad del recurso está clasificada como muy baja con 1000 a 2000 metros cúbicos por habitante al año. Sólo el sureste de México que ha tenido un crecimiento económico poco significativo, ha sido clasificado con una alta disponibilidad de 10000 metros cúbicos por persona al año. Las zonas norte, centro y noreste que contribuyen con el $85 \%$ del Producto Interno Bruto (PIB) y tienen el $77 \%$ de la población sólo cuentan con el 32\% de la disponibilidad de agua, aproximadamente 1874 metros cúbicos por habitante al año. En contraste, la zona sureste que contribuye con un $15 \%$ del PIB y concentra el 23\% de la población, tiene una alta disponibilidad del 66\% de los recursos hidrológicos, aproximadamente 13759 metros cúbicos por persona anuales. De este modo, el promedio de disponibilidad de agua nacional es de 4573 metros cúbicos por individuo al año (Comisión Nacional del Agua, 2012, pp. 14-16).

En el caso de la Zona Metropolitana del Valle de México (ZMVM) habitan 18,620,763 concentrados en un área de 4,979 kilómetros cuadrados y una densidad poblacional de 3,740 personas por kilómetro cuadrado agudizan el servicio intermitente de agua potable como la principal 
Cruz García Lirios, Javier Carreón Guillén, José Marcos Bustos Aguayo,

Jorge Hernández Valdés, José Alfonso Aguilar Fuentes y Francisco Javier Rosas Ferrusca

problemática. En la ZMVM, durante el período que va de 1950 al año 2000 , la población ha aumentado 5.25 veces y pasó de 3'442,557 habitantes a 18'076,572 residentes. Respecto a la densidad poblacional, el Estado de México y el Distrito Federal son la primera y segunda entidades con más viviendas ocupadas y la delegación Iztapalapa es la más poblada con 1.750.336 habitantes, de los cuales la mitad son menores de 15 años (Comisión Nacional de Población, 2008, pp. 23-55).

Tal panorama se exacerba si se considera que la calidad del agua superficial de 393 estaciones en 225 ríos, 81 estaciones en 62 lagos y presas, 26 estaciones en 13 santuarios y sitios costeros, 15 estaciones de descarga de aguas residuales ha sido reportada como muy baja, así como de la subterránea que consta de 228 estaciones en 24 acuíferos, establecidos por el Índice de Calidad del Agua con valores entre 0 y 100 , este último es de valor excelente, después aceptable, levemente contaminada, contaminada, fuertemente contaminada y el último como excesivamente contaminada. El $60.7 \%$ del agua superficial y el $46.3 \%$ de subterránea están contaminadas y fuertemente contaminadas, clasificando al agua superficial del Valle de México como excesivamente contaminada con un 32.49 (Comisión Nacional del Agua, 2005, pp. 13).

El suministro de agua en la ZMVM es de $68 \mathrm{~m} 3 / \mathrm{seg}$, el cual proviene de pozos explotados $25.16 \mathrm{~m} 3 / \mathrm{seg}$. (37\%), pozos recargados $15 \mathrm{~m} 3 / \mathrm{seg}$. (22\%), manantiales $.36 \mathrm{~m} 3 / \mathrm{seg}$. (2\%), del Río Cutzamala $13.6 \mathrm{~m} 3 / \mathrm{seg}$. (20\%) del Río Lerma $6.12 \mathrm{~m} 3 / \mathrm{seg}$. (9\%) y saneamiento con $6.8 \mathrm{~m} 3 / \mathrm{seg}$. (10\%). Respecto al grado de presión del recurso, la ZMVM ocupa el primer lugar con una sobreexplotación del 120\% del agua disponible. A pesar de que la situación hidrológica es extremadamente comprometida en la ZMVM, las demás regiones hidrológicas se encuentran muy cercanas a dicha problemática. Las zonas norte, noreste y centro explotan el 40\% de sus recursos hidrológicos (Comisión Nacional del Agua, 2008, pp. 58-64).

En el caso de la Ciudad de México en 1955 tenía una disponibilidad de 11500 metros cúbicos anuales per cápita. En el año 2004, disminuyó a 4094 metros cúbicos anuales per cápita. En ese mismo año se consumió el 74 por ciento del total de agua potable suministrada, equivalente a 16.157 metros cúbicos por segundo. El agua en el Distrito Federal está destinada a la industria $(17 \%)$, el comercio $(16 \%)$ y uso doméstico (67\%), el cual se divide en el uso de excusado (40\%), regadera $(30 \%)$, ropa $(15 \%)$, trastes $(6 \%)$, cocina $(5 \%)$ y otros $(4 \%)$. 
Iztapalapa al concentrar la mayor población obtuvo el mayor consumo con 2.732 metros cúbicos por segundo equivalentes al 16.9 por ciento del total. Gustavo A. Madero y Álvaro Obregón con 13.75 y 9.94 por ciento respectivamente. En contraste, las delegaciones con menor consumo fueron Cuajimalpa, Tláhuac y Milpa Alta con un 5.97 por ciento. En este sentido, se espera para el 2020 una disponibilidad de 3500 metros cúbicos anuales per cápita. Por ello, la cobertura del servicio es excluyente con 905000 personas que no cuentan con agua potable porque hay una escasez de seis metros cúbicos por segundo (Comisión Nacional del Agua, 2012, pp. 30-36.

En el caso del consumo doméstico de agua, los criterios para establecer la escasez son:

- Crítica entre 1000 y 1700 metros cúbicos anuales per cápita

- Baja entre 1700 y 5000 metros cúbicos anuales per cápita

- Media entre 5000 y 10000 metros cúbicos anuales per cápita

- $\quad$ Alta más de 10000 metros cúbicos anuales per cápita

El 32.27 por ciento de los usuarios se encuentra dentro del umbral del rango, el 78.5 por ciento tiene un consumo menor a 50 metros cúbicos, el 11 por ciento consume menos de 10 metros cúbicos y 0.38 consume más de 180 metros cúbicos bimestrales. El pago promedio en la Ciudad de México fue 110,25 pesos bimestrales. Esto significa una recaudación por cobro de derechos a los usuarios del 80 por ciento en relación con su costo real por el servicio (Comisión Nacional del Agua, 2005, p. 31).

Se estima que en el 2025, el $80 \%$ de la población mundial estará en alta escasez. El pronóstico para el año 2050, implica un rango de aumento de la temperatura de 1,4 a 5,6 centígrados causando un incremento de 44 centímetros del nivel del mar, un 5\% más de las precipitaciones y la extinción de una cuarta parte de las especies. En este sentido, se estima para el año 2025 una crisis mundial de abasto irregular e insalubre de agua en la que 2000 millones de individuos no dispondrán de agua bebible. En el año 2030, el crecimiento poblacional de la Zona Metropolitana del Valle de México (ZMVM) será de 22.5 millones de habitantes, la Zona Metropolitana de Guadalajara (ZMG) de 4.8 millones de habitantes y la Zona Metropolitana de Monterrey (ZMM) de 4.9 millones de habitantes. La problemática de distribución de los recursos 
hídricos se enfocaría en aquellas ciudades con una población mayor a los 500 mil habitantes. Si se consideran las proyecciones poblacionales, las zonas metropolitanas de Guadalajara, Monterrey, Cuernavaca, Tlaxcala, Veracruz, Puebla, Aguascalientes, Toluca, San Luis y Cancún estarían en una crisis de disponibilidad hídrica esperada para el año 2025 (Comisión Nacional del Agua, 2008, pp. 12-15).

El enfoque económico ha establecido un sistema de cobro para reducir el desbalance entre disponibilidad hídrica y consumo de agua, en consecuencia:

- $\quad$ Tarifa por estándar. La tasa por unidad de agua es independiente de la cantidad de agua consumida. La tarifa es la misma sin importar la disponibilidad o el consumo de agua (García, 2010).

- $\quad$ Tarifa por volumen. El precio unitario del agua depende de la cantidad que se utiliza, empero se incrementa o disminuye a partir de la discrecionalidad gubernamental (Jiménez, 2010).

- $\quad$ Tarifa por situación. La tasa por unidad de agua aumenta su costo durante el día y disminuye su costo durante la noche. Durante la temporada de estío se incrementa su costo y durante la temporada de lluvias disminuye su precio unitario (Leff, 2011).

- $\quad$ Tarifa por intervalos. El precio unitario del agua se incrementa en función del volumen consumido. A partir de los intervalos de consumo, se aplican precios que se incrementan conforme el consumo sobrepasa los umbrales permitidos (León, 2013).

- $\quad$ Tarifas por umbrales. El precio unitario del agua es constante en tanto no rebase el umbral de confort. Una vez rebasado el consumo asignado, se aplica un incremento logarítmico (Lucca, 2010).

- $\quad$ Tarifa por autofinanciamiento. El costo unitario del servicio se establece a partir del ingreso familiar y un umbral de confort. Una vez rebasado el límite permitido, se incrementa el costo por cada volumen cúbico extra (Machado, 2012).

- $\quad$ Tarifa por subsidio. El costo unitario del servicio de agua potable implica una cuota estándar o estratificada y un subsidio en función de un umbral de confort (Malmod, 2011).

El enfoque económico sostiene que la disponibilidad de recursos es un indicador de la huella ecológica, la cual puede ser reducida a partir de un balance de costos y beneficios. A medida que los recursos 
naturales escasean, el incremento de tarifas correspondientes orientaría el consumo de otros recursos con mayor disponibilidad. Mientras tanto, el recurso natural en vías de extinción podría conservarse ya que estaría protegido por el alto costo que supone su consumo, empero el consumo de un recurso, desde la aproximación psicosocial, es determinado por procesos de influencia social. Cuando menos, la psicología de la sustentabilidad ha establecido dos procesos de influencia, una de orden mayoritario o directo y otra minoritaria o indirecta.

En contraste, desde la aproximación psicosocial, la influencia mayoritaria plantea que el consumo sistemático de un recurso está determinado por el poder de decisión de la mayoría. Si el grueso de la población tiene por costumbre el aseo personal diario, entonces el individuo se verá influido a adoptar un estilo de vida antropocéntrico en el que los recursos hídricos son considerados como un servicio exclusivo para las necesidades humanas actuales sin importar las capacidades de las generaciones humanas posteriores así como las necesidades de las especies actuales o futuras. El modelo mayoritario es directo porque a través de una fuente considerada experta puede influir sobre la decisión de consumo del individuo. En efecto, la conformidad del individuo es el resultado final de la influencia mayoritaria (Markowitz, 2012).

En contraste, la influencia minoritaria sostiene que el consumo de los recursos naturales obedece a la identidad que establece el individuo para con los grupos que le rodean. De este modo, el aseo personal puede variar según el estilo de vida grupal en el que el individuo está inserto. Si el grupo tiene por norma el aseo personal con un mínimo de agua, entonces el individuo llevará a cabo dicha acción sin importar la disponibilidad de agua. Se trata de una influencia indirecta ya que impacta el estilo de vida futuro más que la decisión de consumo en el presente. Por ello, la innovación es la consecuencia principal de la influencia minoritaria (McCright, 2010).

Ambos procesos de influencia social, mayoritario o minoritario parecen obviar la disponibilidad de los recursos que el enfoque económico muestra como factor esencial, empero son relevantes porque advierten que sin importar la cantidad de agua consumible, la toma de decisión presente o futura está determinada por la norma social o por la norma grupal (Moreno, 2013). 
Cruz García Lirios, Javier Carreón Guillén, José Marcos Bustos Aguayo,

Jorge Hernández Valdés, José Alfonso Aguilar Fuentes y Francisco Javier Rosas Ferrusca

\section{Los estudios relativos a las correlaciones entre determinantes del consumo de agua}

Los EPS pueden ser analizados desde una lógica de centralidad y periferia. A partir de esta nomenclatura, es posible advertir que el Desarrollo Sustentable es un tema o nodo central que implica al cambio climático, el calentamiento global, el efecto invernadero y las emisiones de carbono como los factores ambientales que tienen un impacto directo sobre la calidad del aire y la salud de las vías respiratorias en las economías y ciudades económicamente desarrolladas, pero además de la contaminación atmosférica, las problemáticas hídricas y las de los residuos municipales son temas centrales en la periferia económica y urbana debido a que los recursos naturales del hemisferio sur son transformados en satisfactores para el hemisferio norte como es el caso del petróleo crudo y sus derivados (Abramo, 2012).

En este sistema de centralidad y periferia, la psicología de la sustentabilidad parece estar dividida en dos vertientes en la que la psicología del sur trata de comprender y explorar los saberes y racionalidades, espacios y riesgos que derivan del impacto de la explotación y transformación de la naturaleza sobre los estilos de vida de las comunidades (Acosta, 2010).

Por su parte, la psicología de la sustentabilidad en el hemisferio norte está más avocada a describir y explicar los efectos del cambio climático sobre las redes de gestión, innovación y emprendimiento que se desarrollan en las economías desarrolladas en referencia a las economías emergentes.

En el hemisferio norte, la psicología de la sustentabilidad inició la descripción de la calidad del entorno y la conciencia ambiental para arribar al estudio de trayectorias y estructuras de variables en modelos de ecuaciones a fin de predecir comportamientos desfavorables o vinculatorios con la sustentabilidad, equidad y felicidad (Behancourth, 2010).

Los modelos de ecuaciones estructurales, trayectorias, estructuras y disturbios tuvieron sus antecedentes en estudios de correlación y regresión a partir de los cuales se establecieron las asociaciones que permitieron la modelación de relaciones de dependencia entre variables (Blunda, 2010).

A pesar de que los modelos de ecuaciones estructurales tienen su fundamento en la covarianzas, las correlaciones y regresiones permitieron 
la especificación de modelos (Carosio, 2010). Por ello, en un escenario de desarrollo sustentable, los agentes en torno al nodo central de conocimiento interactúan para dar forma a un sistema en equilibrio donde la centralidad depende de la periferia, el norte del sur, el este del oeste.

Sin embargo, el estado del conocimiento tiende a configurar una red descriptiva de las problemáticas medioambientales, aunque los estudios también se orientan a la explicación de trayectorias y estructuras en las que los temas son integrados en modelos a fin de poder anticipar los efectos de las problemáticas en la psique y el comportamiento.

De este modo, una revisión de los estudios psicológicos de la sustentabilidad de 2010 a 2014 muestra que los valores, percepciones y creencias son las variables determinantes del consumo (véase tabla 1 en anexo). En este sentido, las tres variables son consideradas exógenas a las actitudes, intenciones, competencias y uso (Corral, 2010).

Los valores implican relaciones de interdependencia entre la naturaleza y las comunidades (biosferismo), relaciones de arraigo entre los grupos en función de la diversidad ecosistémica (comunitarismo), relaciones de competencia entre los seres humanos (indivualismo) en función de la escasez de recursos y relaciones de equilibrio entre las generaciones (sostenibilismo) en función de la austeridad de la humanidad actual, las tecnologías futuras y la disponibilidad de los recursos (Nozica, 2011).

Las percepciones denotan la exposición involuntaria al riesgo, la ausencia de un control de la situación (incertidumbre) y el escepticismo a la información generada por instituciones de protección civil (Quiroz, 2013). En este sentido, la percepción hacia situaciones de riesgos normales y extraños se representa explícitamente a partir de experiencias e información no experimentada (Sharples, 2010). Por consiguiente, implica indicación de peligro, prevención, contingencia, manejo y protección; expectación que determina una acción y reacción de solución rápida (Barkin y Lemus, 2011). Se pueden definir como una respuesta inmediata y simplificada a los peligros y las incertidumbres que determinan juicios, decisiones y conductas (Bertoni y López, 2010).

Las creencias son planteadas como desorientadoras (paradigma social dominante, paradigma de la excepción humana, antropocentrismo, materialismo, progresismo y utilitarismo) y como orientadoras (nuevo paradigma ambiental, conservadurismo, ecocentrismo, naturalismo $\mathrm{y}$ austeridad) de los comportamientos humanos hacia la protección del 
Cruz García Lirios, Javier Carreón Guillén, José Marcos Bustos Aguayo,

Jorge Hernández Valdés, José Alfonso Aguilar Fuentes y Francisco Javier Rosas Ferrusca

medio ambiente (Corral y Domínguez, 2011). Las creencias que impiden el desarrollo sostenible denotan que el comportamiento humano y su crecimiento económico están exentos de las leyes de la naturaleza, por lo tanto, dicho crecimiento sólo esta determinado por el avance tecnológico (Duerden y Witt, 2010). En contraste, las creencias que favorecen el desarrollo sostenible implican el replanteamiento de las visiones antropocéntricas, el establecimiento de los límites al crecimiento económico, la importancia del equilibrio ecológico, el desarrollo sostenible necesario (Flores y Parra, 2011). Las creencias en torno a la supremacía de las necesidades humanas sobre los procesos de la naturaleza, la consecuente concepción del equilibrio o desequilibrio de las necesidades humanas con los procesos de la naturaleza y consiguiente crecimiento económico ilimitado o limitado, se presentan con un grado diferente íntercultural, económica y generacionalmente (García y Corral, 2010).

Los EPS plantean relaciones significativas entre las variables culturales (valores) y las variables ideológicas (creencias). Es decir, se considera que tanto la cultura como la ideología influyen en el individuo a través de los valores y las creencias que se amplifican en los discursos de la gente y que el individuo capta, aprende y reproduce ante una situación específica (Gissi y Soto, 2010). En este sentido, el abasto irregular de agua característico de las ciudades modernas y las urbes periféricas está asociado a valores y creencias en torno a su disponibilidad exclusiva para el consumo humano o su disponibilidad compartida entre las especies (Hernández y Jiménez, 2010).

Las asociaciones endógenas develan las diferencias intra e interculturales e ideológicas. Las sociedades colectivistas tales como las asiáticas, latinas y europeas del este se caracterizan por valores biosféricos-altruistas y creencias ecocéntricas que favorecen el cuidado del medio ambiente al ser considerado como su hábitat y las especies como sus compañeras hermanas de coexistencia (Hidalgo y Pisano, 2010). En torno a la insalubridad por la infraestructura hidrológica deficiente o inexistente, las comunidades y los barrios populares se solidarizan para el autocuidado de los niños (Izasa y Enao, 2010).

En la medida en que la insalubridad aumenta, la solidaridad comunitaria también se incrementa. Las sociedades europeas y norteamericanas, en contraste, se caracterizan por valores individualistas y creencias antropocéntricas. Incluso en los grupos migrantes colectivistas que residen en estas sociedades se observa un cambio de valores y 
creencias que los acerca a individualismo y al antropocentrismo (Jaén y Barbudo, 2010). La disponibilidad de agua, asociada a los valores de sobreexplotación y las creencias de abundancia del recurso, orienta la elaboración de un modelo en el que se evidencien el aumento de las dos variables culturales e ideológicas en la medida en que se incrementa la información sobre la abundancia de agua (Kalantari y Asadi, 2010).

La influencia de la estructura social individualista y antropocéntrica también se observa en los países con economías emergentes (Brasil, Rusia, India, China) que serán desarrolladas en la década que se aproxima. Se trata de economías que transitan del colectivismo al individualismo, del biosferismo al industrialismo, del ecocetrismo al antropocentrismo (Londoño y Cardona, 2011). El crecimiento económico está asociado con la inversión pública en infraestructura hidrológica. Los proyectos energéticos e hidrológicos están correlacionados con las necesidades de las urbes. La inversión en torno al servicio público del agua está asociado al incremento de la población en las ciudades, sus dimensiones, servicios y migración (Manríquez y Montero, 2011). El consumo de agua registra un incremento en sus tarifas asociadas con la escasez en los barrios periféricos (Martínez y Montero, 2011). El desabasto de agua vinculada con la insalubridad e implicada con las epidemias aumenta las muertes infantiles.

Son las relaciones entre las variables espaciales (diseños), las variables económicas (riesgo y utilidad), las variables educativas (conocimientos) y las variables individuales (actitudes, habilidades, intenciones, comportamientos).

Los estudios en torno a la influencia de las edificaciones sobre la percepción individual demostraron que la estética, la funcionalidad y el diseño tienen un efecto directo, positivo y significativo sobre la satisfacción del cliente (McCright y Dunlap, 2011). Después, los estudios vinculados con la influencia de las masas al interior de edificaciones sobre el comportamiento humano demostraron que el hacinamiento, el ruido o la densidad son factores que determinan el estrés del cliente (Milfont y Duckitt, 2010). Finalmente, los estudios relacionados con la influencia de los eventos al interior de edificaciones sobre la cognición individual demostraron que las personas se forman actitudes hacia los eventos, edificios y espectadores (Montalbetti y Chamarro, 2010). Las edificaciones vinculadas con la bioseguridad hidrológica (reservas de agua potable) demuestran la relevancia de las políticas sanitarias, las contingencias 
epidémicas, las catástrofes pandémicas, la competencia por los recursos y la solidaridad comunitaria (Montalvo y Chábves, 2011).

Las asociaciones endógenas develan impactos diferentes de los espacios, los ingresos, los conocimientos y las actitudes sobre los comportamientos.

En las economías industriales con políticas neoliberales, los comportamientos contaminantes han sido asociados con las percepciones utilitarias, las actitudes racionales y los conocimientos tecnológicos (Touguinha y Pato, 2011). Los proyectos hidrológicos están diseñados para incrementar la utilidad personal más que la utilidad social. Es decir, el servicio de agua potable sólo está disponible para aquellas zonas que pueden pagar el costo del servicio (García, 2014). En las economías postindustriales con políticas sociales, los comportamientos preservadores han sido vinculados con las percepciones de riesgo, las actitudes afectivas y los conocimientos sociales. Los proyectos hidrológicos están vinculados con los servicios de todo tipo. Se trata de abastecer a las zonas comerciales relacionadas con el turismo (Zapata y Castrechini, 2011).

En las economías informacionales con políticas sostenibles, los comportamientos ecológicos han sido vinculados con las percepciones de responsabilidad, las actitudes globales y los conocimientos organizacionales. Los proyectos hidrológicos se enlazan con la normatividad sostenible que obliga una disponibilidad hidrológica equitativa entre las zonas y las especies (García, 2012).

A partir de los estudios asociativos, tanto exógenos como endógenos, se han abstraído estructuras económicas, políticas y sociales que influyen en los individuos (Leff, 2010). Los análisis de correlación evidencian los modelos de consumo que culpan a los individuos del deterioro global y platean el accionar aislado como la solución al problema global. Ante la escasez, desabasto e insalubridad ambientales se plantea que la educación ambiental es la acción indicada para prevenir dichas situaciones y las ecotasas (multas e incentivos) son las estrategias fiscales efectivas para el desarrollo sostenible (García, 2011).

Las asociaciones, tanto exógenas como endógenas, orientan el diseño de modelos teóricos estructurales. Una relación causal entre una variable $\mathrm{X}$ y una variable $\mathrm{Y}$ subyace de una asociación exógena entre una variable $\mathrm{W}$ y una variable $\mathrm{X}$. $\mathrm{O}$ bien, los determinantes de una variable $\mathrm{Z}$ subyacen de las asociaciones entre $\mathrm{W}, \mathrm{X}$ y Y. Es decir, a partir de las asociaciones se infieren las relaciones causales. Si existe una 
asociación significativa entre las variables independientes puede haber relaciones causales entre ellas. Si existen asociaciones espurias entre las variables independientes puede haber relaciones causales con una variable dependiente. Una correlación positiva y significativa entre la escasez, el desabasto y la insalubridad ambiental permite la elaboración de un modelo en el que el ahorro de agua está determinado por las tres situaciones ambientales. Una correlación negativa y significativa entre las tres variables permite un diseño en el que el dispendio de agua es el efecto esperado. Una correlación espuria entre las tres situaciones ambientales orienta el diseño de un modelo en el que otras variables situaciones estarían explicando el dispendio o el ahorro de agua.

\section{Discusión}

Este trabajo ha establecido diferencias entre políticas públicas de abastecimiento y cobro del servicio municipal de agua a partir de contrastar dos enfoques: uno económico que pondera el costo real a partir de la oferta y demanda y otro psicológico que establecería un precio unitario del agua a partir de la relación entre usuarios y autoridades.

En ese sentido, los estilos de vida antropocéntricos que corresponden a grupos con creencias relativas a la abundancia de agua corresponden a acciones de dispendio, en contraste, los estilos de vida ecoperiféricos corresponderían a grupos que creen en una sequía generalizada del agua y en consecuencia optimizan y reutilizan el recurso.

Sin embargo, los estudios relativos al enfoque económico y a la aproximación psicosocial parecen indicar que el incremento del precio unitario del agua provoca una reducción significativa en el consumo del recurso, empero la relación entre autoridades y usuarios respecto al abastecimiento parece estar determinada por fenómenos sociopolíticos electorales.

Por consiguiente, la relación entre disponibilidad y consumo ex-

plicaría en menor medida los conflictos derivados de una política de tandeo, incremento de tarifas o reducción de subsidios.

Incluso, los estilos de vida al interior de los barrios o comunidades tampoco anticiparían posibles conflictos entre usuarios y autoridades tales como secuestros de pipas, boicots al suministro, cierres de avenidas o confrontaciones con la policía. 
Son más bien las coyunturas sociopolíticas, contiendas electorales y movilizaciones ciudadanas las que determinarían la exacerbación de posturas entre gobernantes y gobernados.

Por lo tanto, es menester revisar los estudios relativos a la acción colectiva y la movilización social en coyunturas pre y post electorales a fin de poder establecer los factores que restablecerían la paz pública, o bien, exacerbarían las diferencias entre los actores y agentes implicados en el desabastecimiento de agua.

En referencia a las revisiones de García (2010; 2011; 2012; 2013 ; 2014) en las que se observa un incremento sustancial de los conflictos entre autoridades y usuarios a partir del incremento de tarifas y la reducción de subsidios, así como la emergencia de estilos de vida austeros, el presente trabajo advierte que la problemática ha transitado por tres periodos en torno a los cuales la disponibilidad y el consumo registraron un desbalance que se exacerbó con el incremento de la densidad poblacional. Posteriormente, en una segunda fase de la problemática, las políticas públicas se orientaron al abastecer a las multinacionales y fraccionamientos residenciales en detrimento de los pueblos originarios, sus usos y costumbres. En este periodo, la influencia social, la cooperación y la solidaridad entre las comunidades derivó en conflictos por la administración de los recursos comunes. Por último, en el tercer periodo las variables sociopolíticas de ordenamiento territorial, así como las subvenciones derivaron en políticas de tandeo que exacerbaron los desencuentros entre los actores políticos, agentes económicos y esferas civiles.

\section{Conclusiones}

El aporte de este trabajo al estado del conocimiento estriba en comparar dos aproximaciones antagónicas que, sin embargo, fueron rebasadas por las políticas de tandeo, subsidio y tarifaria que se implementaron en las urbes ante el crecimiento de la demanda inmobiliaria y el aumento del precio unitario del servicio hídrico. En este escenario, el presente escrito anticipa conflictos derivados de las coyunturas electorales más que de las diferencias en los estilos de vida antropocéntricos o ecoperiféricos, así como entre la disponibilidad de agua y consumo humano. 


\section{Referencias bibliográficas}

Abramo, P. (2012). La ciudad com-fusa: mercado y producción de la estructura urbana en las grandes metrópolis latinoamericanas. Eure, 38 (114) 35-69.

Acosta, A. (2010). Sólo imaginando otros mundos, se cambiará este. Reflexiones sobre el buen vivir. Sustentabilidades, 2, 5-21.

Barkin, D. y Lemus, B. (2011). La economía ecológica solidaria. Una propuesta frente a nuestra crisis. Sustentabilidades, 5, 4-10.

Behancourth, L. (2010). Los consumidores ecológicos y el fomento de los mercados verdes; una alternativa hacia el bienestar espíritu, mente y salud a partir de la adopción de estilos de vida saludables. Eleuthera, 4, 193-210.

Bertoni, M. y López, M. (2010). Valores y actitudes hacia la conservación de la reserva de la biosfera. Estudios y Perspectivas de Turismo, 19, 835-849.

Blunda, Y. (2010). Percepción de riesgo volcánico y conocimiento de los planes de emergencia en los alrededores del volcán Poas, Costa Rica. Revista Geológica de América Central, 43, 201-209.

Carosio, A. (2010). La cultura del consumo contra la sustentabilidad de la vida. Sustentabilidades, 2, 39-52.

Comisión Nacional del Agua (2005). Estadísticas del agua en México. México: Conagua.

Comisión Nacional del Agua (2008). Programa Hídrico Nacional. 2007-2012. México: Conagua.

Comisión Nacional del Agua (2012). Bancos de agua en México. México: Conagua.

Corral, V. (2010). Psicología de la sustentabilidad. Un análisis de lo que nos hace proecológicos y prosociales. México: Trillas.

Corral, V. y Domínguez, R. (2011). El rol de los eventos antecedentes y consecuentes en la conducta sustentable. Revista Mexicana de Análisis de la Conducta, 37, 9-29.

Duerden, M. y Witt, P. (2010). The impact of direct and indirect experiences on the development of environmental knowledge, attitudes and behavior. [El impacto de las experiencias directas e indirectas en el desarrollo de los conocimientos medioambientales, actitudes y comportamientos] Journal of Environmental Psychology, 30, 379-392. 
Flores, M. y Parra, M. (2011). Caracterización del ahorro doméstico de agua en la región de Murcia en función de componentes sociodemográficos. Contribuciones a las Ciencias Sociales, 13, 1-13.

García, C. y Corral, V. (2010). La identidad social y el locus de control en habitantes pobres del sur de Nuevo León, México. Revista de Psicología Socia, 25, 231-239.

García, C. (2010). La exclusión hidrológica. Revista Interdisciplinar Entelequia, 11, 41-59.

García, C. (2011). Teorías psicosociales para explicar los conflictos derivados del abastecimiento de agua en México, Distrito Federal. Revista Pampedia, 8, 56-68.

García, C. (2012). Los estilos de vida en torno a las problemáticas hídricas. Sustentabilidades, 7, 84-92.

García, C. (2013). Los estudios psicológicos de la sustentabilidad hídrica. Aplicaciones al sistema tarifario de consumo. Revista de Ciencias Sociales, 139, 65-90.

García, C. (2014). Teoría de la actitud hacia el consumo sustentable de agua. Sustentabilidades, 8, 33-41.

Gissi, N. y Soto, P. (2010). De la estigmatización al orgullo barrial: Apropiación del espacio e integración social de la población mixteca en una colonia de la Ciudad de México. INVI. 68, 99-118.

Hernández, L. y Jiménez, E. (2010). Actitudes y comportamiento ambiental del personal de área de conservación marina. Biocenosi, 23, 1-12.

Hidalgo, C. y Pisano, I. (2010). Predictores de la percepción de riesgo y del comportamiento ante el cambio climático. Un estudio piloto. Psyechology, 1, 36-49

Instituto Nacional de Estadística Geografía e Informatica (2010). Mujeres y hombres en México. México: Inegi.

Izasa, L. y Enao, G. (2010). El desempeño en habilidades sociales en niños, de dos y tres años de edad, y su relación con los estilos de interacción parental. Journal of Research in Educational Psycho$\log , 8,1051-1076$.

Jaén, J. y Barbudo, P. (2010). Evolución de las percepciones medioambientales de los alumnos de educación secundaria en un curso académico. Revista Eureka, Enseñanza e Investigación Cientifi$c a, 7,247-259$. 
Jiménez, M. (2010). Definición y medición de la conciencia ambiental. Revista Internacional de Sociología, 68, 735-755.

Kalantari, K. y Asadi, A. (2010). Designing a structural model for explained environmental attitude and behavior of urban residents. [Diseño de un modelo estructural para explicar la actitud ambiental y el comportamiento de los residentes urbanos]. International Journal for Environmental Research, 4, 309-320.

Leff, E. (2011). Sustentabilidad y racionalidad ambiental: hacia "otro" programa de "sociología ambiental". Revista Mexicana de Sociología, 73, 5-46.

Leff, E. (2010). Economía ecológica, racionalidad y sustentabilidad. Sustentabilidades, 2, 106-119.

León, S. (2013). Indicadores de tercera generación para cuantificar la sustentabilidad urbana ¿Avances o estancamiento? EURE, 39, (118), 173-198.

Londoño, C. y Cardona, H. (2011). Estado del arte de los recursos para el desarrollo. Revista Ciencias Estratégicas, 19, 35-54.

Lucca, E. (2010). Sustentabilidad urbana, rural natural. Sustentabilidades, 2, 120-142.

Machado, C. (2012). Aproximaciones para la reestructuración física y social de la vivienda popular de Caracas. En Teolinda, Bolivar. y Erazo, Jaime (coord.), Dimensiones del habitad popular mexicano, (pp. 337-352). Quito: Clacso.

Malmod, A. (2011). Lógicas de ocupación en la conformación del territorio. Ordenamiento territorial como instrumento de la planificación. Revista Iberoamericana de Urbanismo, 6, 18-30.

Manríquez, J. y Montero, M. (2011). Motivación hacia el cuidado del agua en población mexicana. Quaderns de Psicología, 13, 25-34.

Markowitz, E. (2012). Is climate change and ethical issue? Examining young adult's beliefs about climate and morality. [¿Es el cambio climatico un asunto ético? Examinando las creencias de adultos en torno al clima y la moralidad]. Climate Chngea, 1, 1-19.

Martínez, J. y Montero, M. (2011). La percepción de restauración ambiental de la vivienda y el funcionamiento familiar. Quaderns de Psicología, 13, 81-89.

McCright, A. (2010). The effects of gender of climate change knowledge and concern in the American public. [Los efectos sobre el 
género del conocimiento en torno al cambio climático y las audiencias en América]. Population and Environment, 32, 66-87.

McCright, A. y Dunlap, R. (2011). Cool dudes: the denial of climate change among conservative white males in the United States. [Actores fríos: la negación del cambio climático entre los hombres blancos conservadores de Estados Unidos]. Global Environmental Change, 1, 1-10.

Milfont, T. y Duckitt, J. (2010). The environmental attitudes inventory: a valid and reliable measure to assess the structure of environmental attitudes. [Inventario de Actitud Ecológica: una medida válida y fiable para evaluar la estructura de las actitudes ambientales]. Journal of Environmental Psychology, 30, 80-94.

Montalbetti, T. y Chamarro, A. (2010). Construcción y validación del cuestionario de percepción de riesgo en escalada de roca. Cuadernos de Psicología del Deporte, 10, 43-56

Montalvo, R. y Chábves, M. (2011). La reignificación del espacio y la identidad genérica en la región agrícola del Tepeyanco, Tlaxcala. En A. Conde, Ortiz, P. y Delgado, A. (coord.), El medio ambiente como sistema socio ambiental. Reflexiones en torno a la relación humanos naturaleza, (pp. 143-176). Tlaxcala: UAT.

Moreno, M. (2013). Una lectura prospectiva de la agenda Rio+20. La emergencia de una gobernanza para el Desarrollo Sustentable. Xihmai, 15 (8) 57-74.

Nozica, G. (2011). Planificar para la integración territorial. Los escenarios deseables de inserción de la provincia de San Juan al Mercosur. Revista Iberoamericana de Urbanismo, 6, 43-54.

Quiroz, D. (2013). Las ciudades y el cambio climático: el caso de la política climática de la ciudad de México. Estudios Demográficos y Urbanos, 28 (83), 343-382.

Sharples, D. (2010). Communicating climate science: evaluating the UK public's attitude to climate change. [Comunicar la ciencia del clima: la evaluación de la actitud del público del Reino Unido ante el cambio climático]. Earth and Environment, 5, 185-205.

Touguinha, S. y Pato, C. (2011). Valores personales, creencias ambientales ecocéntricas y comportamiento ecológico de trabajadores brasileños: el caso del ministerio público del Distrito Federal y territorios. Quaderns de Psicología, 13, 35-45. 
United Nations Habitad (2010). Sick water? The central role of wastewater management in sustainable. A rapid response assessment. [El agua enferma? El papel central de la gestión de aguas residuales en sostenible. Una evaluación de respuesta rápida]. Birkiland: UN-Habitad

United Nations Water (2013). Water security \& the global water agenda. [Seguridad hídrica y la agenda mundial del agua]. Ontario: United Nations University

Zapata, R. y Castrechini, A. (2011). Conducta Proambiental y personalidad: Análisis de un barrio de Lima. Quaderns de Psicología, 13, 47-61. 


\section{APPËNDICE}

\section{Tabla 1. Estado del conocimiento}

\begin{tabular}{|c|c|c|c|}
\hline Año & Autor & Muestra & Hallazgos \\
\hline 2010 & McCright & $\begin{aligned} 1060 & \rightarrow 2001 ; 1006 \rightarrow 2002 ; \\
1003 \rightarrow 2003 ; 1005 & \rightarrow 2004 ; \\
1004 & \rightarrow 2005 ; 1000 \rightarrow 2006 ; \\
1009 & \rightarrow 2007 ; 1012 \rightarrow 2008\end{aligned}$ & $\begin{array}{l}\text { La ideología política y percepción de com- } \\
\text { prensión determinó negativamente al cono- } \\
\text { cimiento sobre el cambio climático y la preo- } \\
\text { cupación por sus consecuencias en el género } \\
(\beta=-0,372 \text { y } \beta=0,336 \text { respectivamente). }\end{array}$ \\
\hline 2010 & Sharples & Grupo focal de docentes & $\begin{array}{l}\text { La fuente principal de información sobre } \\
\text { el cambio climático fueron los noticia- } \\
\text { rios de televisión ( } 23,9 \%) \text {, los alimentos } \\
\text { y bebidas con los más consumidos por la } \\
\text { muestra }(83,8 \%) \text {, los focos fueron el obje- } \\
\text { to más utilizado para combatir el cambio } \\
\text { climático }(88,7 \%) \text {, . }\end{array}$ \\
\hline 2010 & $\begin{array}{l}\text { Hidalgo y } \\
\text { Pisano }\end{array}$ & 84 estudiantes universitarios & $\begin{array}{l}\text { La actitud se relacionó con los conoci- } \\
\text { mientos }(\mathrm{r}=0,454, \mathrm{p}=0,001) \text {, la auto- } \\
\text { eficacia con los conocimientos y las ac- } \\
\text { titudes }(\mathrm{r}=0,303 \mathrm{y} \mathrm{r}=0,882 ; \mathrm{p}=0,001 \\
\text { respectivamente), la percepción de riesgo } \\
\text { con el conocimiento, actitud y autoefi- } \\
\text { cacia }(\mathrm{r}=0,475 ; \mathrm{r}=0,589 ; \mathrm{r}=0,547 ; \mathrm{p} \\
=0,001 \text { respectivamente), la intención } \\
\text { con el conocimiento, actitud, autoefica- } \\
\text { cia, percepción e intención }(\mathrm{r}=0,206 ; \mathrm{r} \\
=0,317 ; \mathrm{r}=0,390, \mathrm{r}=0,382 ; \mathrm{p}=0,001 \\
\text { respectivamente). } \\
\text { La percepción de riesgo fue determinada } \\
\text { por la actitud }(\beta=0,305 ; \mathrm{p}=0,000) \text { y la } \\
\text { intención fue influida por la autoeficacia } \\
(\beta=0,259 ; \mathrm{p}=0,001) \text {. }\end{array}$ \\
\hline 2010 & Jiménez & 1305 Residentes andaluces & $\begin{array}{l}\text { Establecieron tres factores de las cuatro } \\
\text { dimensiones posibles. El primer factor } \\
\text { explicó el } 46,4 \% \text { de la varianza mientras } \\
\text { que el segunda factor explicó el } 28,6 \% \\
\text { de la varianza y el tercer factor explicó el } \\
25,15 \text { de la varianza. } \\
\text { Establecieron diferencias entre hombres y } \\
\text { mujeres [X2 }=10,088(2 \mathrm{gl}) \mathrm{p}=0,007] \\
\text { por años [X2 }=176,77(8 \mathrm{gl}) \mathrm{p}=0,000] \\
\text { y habitad }[\mathrm{X} 2=21,657(6 \mathrm{gl}) \mathrm{p}=0,001]\end{array}$ \\
\hline 2010 & $\begin{array}{l}\text { Gissi y } \\
\text { Soto }\end{array}$ & $\begin{array}{l}\text { Migrantes mixtecos en San } \\
\text { Miguel Teoitongo, México, } \\
\text { Distrito Federal }\end{array}$ & $\begin{array}{l}\text { La apropiación del espacio se realiza a } \\
\text { partir del tequio que es el trabajo personal } \\
\text { que realiza un integrante antes de entrar a } \\
\text { la guatza o trabajo comunitario. }\end{array}$ \\
\hline
\end{tabular}




\begin{tabular}{|c|c|c|c|}
\hline Año & Autor & Muestra & Hallazgos \\
\hline 2010 & $\begin{array}{l}\text { Molini y } \\
\text { Salgado }\end{array}$ & $\begin{array}{l}\text { Documentos de políticas } \\
\text { públicas y gestión municipal }\end{array}$ & $\begin{array}{l}\text { En torno a la discusión de las diferencias } \\
\text { entre ciudad compacta y dispersa, la den- } \\
\text { sidad poblacional es un factor relevante ya } \\
\text { que la baja concentración en la ciudades } \\
\text { compactas la hacen más sustentable que } \\
\text { las ciudades dispersas, pero su alta den- } \\
\text { sidad incrementa los costos a la entidad } \\
\text { gubernamental encargada de regularla. } \\
\text { Tal panorama afecta la creación de unifa- } \\
\text { miliares porque éstas se producen en co- } \\
\text { munidades con más de } 500 \text { mil habitantes } \\
\text { y presionan la disponibilidad de recursos. }\end{array}$ \\
\hline 2011 & $\begin{array}{l}\text { McCright } \\
\text { y Dunlap }\end{array}$ & $\begin{array}{l}1000 \text { a } 1060 \text { encuestados de } \\
2000 \text { a } 2010\end{array}$ & $\begin{array}{l}\text { Las creencias en torno a los efectos nulos } \\
\text { del cambio climático determinaron la con- } \\
\text { fianza en los hombres blancos con ideolo- } \\
\text { gía conservadora }(\gamma=0,82 ; p=0,000) \text {. } \\
\text { Por su parte la ideología política de base } \\
\text { determinó la negación de los efectos del } \\
\text { cambio climático }(\gamma=0,47 ; p=0,000) \text {, } \\
\text { la raza determinó la creencia acerca de la } \\
\text { falta de consenso de los efectos del cam- } \\
\text { bio climático para los blancos conserva- } \\
\text { dores }(\gamma=0,38 ; p=0,000) \text {, } \\
\text { empero, el sexo incidió negativamente } \\
\text { sobre la creencias de los efectos nulos del } \\
\text { cambio climático en los encuestados base } \\
(\gamma=-0,67 ; p=0,000) \text { así como la iden- } \\
\text { tificación con el ambientalismo sobre la } \\
\text { misma creencia en el mismo grupo }(\gamma= \\
-0,81 ; p=0,000) \text {. }\end{array}$ \\
\hline 2011 & $\begin{array}{l}\text { Touginha } \\
\text { y Pato }\end{array}$ & 295 trabajadores brasileños & $\begin{array}{l}\text { El comportamiento ecológico correlacio- } \\
\text { nó con la edad }(\mathrm{r}=0,30) \text { mientras que las } \\
\text { creencias ecocéntricas se relacionaron } \\
\text { con los valores universales }(\mathrm{r}=0,20) \text {. } \\
\text { Por su parte, la edad y los valores uni- } \\
\text { versales determinaron al comportamiento } \\
\text { ecológico }(\beta=0,24 ; \beta=0,21 ; \mathrm{p}=0,001 \\
\text { respectivamente). }\end{array}$ \\
\hline
\end{tabular}




\begin{tabular}{|c|c|c|c|}
\hline Año & Autor & Muestra & Hallazgos \\
\hline 2011 & $\begin{array}{l}\text { Nacif y } \\
\text { Espinosa }\end{array}$ & $\begin{array}{l}\text { Documentos histórico- } \\
\text { urbanísticos }\end{array}$ & $\begin{array}{l}\text { Encontraron una relación entre la identi- } \\
\text { dad nacional y el pragmatismo urbanista } \\
\text { del reordenamiento espacial central y los } \\
\text { diseños arquitectónicos. Las edificaciones } \\
\text { representaron símbolos de reconstrucción } \\
\text { nacional que se extenderían a otras ciu- } \\
\text { dades pamperas y sudamericanas: Brasil, } \\
\text { Perú, Colombia y Venezuela. Las propues- } \\
\text { tas arquitectónicas de la época plantea- } \\
\text { ban una mayor movilidad de este a oeste } \\
\text { tratando de integrar a la periferia con el } \\
\text { centro. De este modo, el campo se articu- } \\
\text { laría con la ciudad y los sistemas hídricos } \\
\text { podrían tener un mayor aprovechamiento, } \\
\text { aunque la región minera se apartaba cada } \\
\text { vez más de los servicios públicos. En tal } \\
\text { esquema, las vías férreas fueron funda- } \\
\text { mentales para incorporar los sectores pri- } \\
\text { marios, secundarios y terciarios. Por ello, } \\
\text { las bodegas debían ser trasladas a las zonas } \\
\text { agroindustriales. Debido a que la ciudad } \\
\text { fue devastada por un sismo y los espacios } \\
\text { de resguardo fueron nulos o insuficientes, } \\
\text { se proyectaron parques recreativos que } \\
\text { cumplieron con la función espacial sísmica } \\
\text { y recreativa. Para evitar el aglutinamiento } \\
\text { de transporte, se proponía la construcción } \\
\text { de un arco. Respecto al reordenamiento } \\
\text { vecinal, la creación de barrios de } 15 \text { man- } \\
\text { zanas en espacios arrendados garantizaba } \\
\text { el control socioespacial del Estado. Otras } \\
\text { propuestas consistieron en concentrar a la } \\
\text { ciudadanía en áreas multiculturales para } \\
\text { evitar la segregación. Dos temas resultaron } \\
\text { fundamentales: la conservación ambiental } \\
\text { y la privatización del territorio. }\end{array}$ \\
\hline 2011 & Malmod & Documentos ilustrativos & $\begin{array}{l}\text { Sistematizó los planes de reordenamiento } \\
\text { a partir de una lógica de exclusión e inclu- } \\
\text { sión. La primera consistió en diferenciar } \\
\text { los espacios; privatización de bienes y ser- } \\
\text { vicios. En contraste, la segunda propuesta } \\
\text { consistió en establecer conexiones entre } \\
\text { los sectores, espacios y servicios para ami- } \\
\text { norar la segregación espacial. La lógica de } \\
\text { inclusión implica un diseño de redes en los } \\
\text { que cada nodo está interconectado el uno } \\
\text { con el otro y permite la interrelación en- } \\
\text { tre los elementos espaciales, así como la } \\
\text { construcción de una identidad urbana que } \\
\text { favorece la tolerancia a la diversidad. }\end{array}$ \\
\hline
\end{tabular}




\begin{tabular}{|c|c|c|c|}
\hline Año & Autor & Muestra & Hallazgos \\
\hline 2011 & Nozica & $\begin{array}{l}\text { Documentos de Política de } \\
\text { Turismo }\end{array}$ & $\begin{array}{l}\text { La política de turismo incentivará la co- } \\
\text { nexión entre corredores bioceánicos y pe- } \\
\text { riurbanos. Para tal propósito, el escenario } \\
\text { deseable consistirá en una red vial que ar- } \\
\text { ticule ambas áreas. Tal estrategia permiti- } \\
\text { rá incrementar las ventajas competitivas } \\
\text { en materia de servicios turísticos, tecno- } \\
\text { lógicos y comerciales de la región. }\end{array}$ \\
\hline 2012 & Markowitz & $606 \rightarrow 2010 ; 316 \rightarrow 2011$ & $\begin{array}{l}\text { Establecieron diferencias entre éticos, no } \\
\text { éticos e indecisos con respecto a su pre- } \\
\text { ocupación }(F=102,52 ; p=0,000) \text {, ries- } \\
\text { gos }(F=51,68 ; p=0,000) \text {, consenso }(F= \\
26,83 ; p=0,000) \text {, eficacia }(F=34,67 ; p \\
=0,000) \text {, responsabilidad }(F=69,41 ; p= \\
0,000) \text {. Las intenciones ambientales fueron } \\
\text { determinadas por las creencias }(\beta=0,506) \text {. }\end{array}$ \\
\hline 2012 & Cravino & 400 residentes bonaerenses & $\begin{array}{l}\text { Encontró un grado de percepción de riesgo } \\
\text { en los residentes bonaerenses al momento } \\
\text { de migrar a la periferia. En este sentido, } \\
\text { la percepción del hábitat está relacionada } \\
\text { con los servicios y las inversiones que el } \\
\text { Estado ha orientado a la centralidad. Otro } \\
\text { factor de percepción de la vivienda es la } \\
\text { socialización espacial ya que un cambio de } \\
\text { barrio implica la pérdida de capital social. } \\
\text { El alquiler es un fenómeno estrechamente } \\
\text { relacionado con las expectativas de apro- } \\
\text { piación del espacio puesto que una buena } \\
\text { raíz garantiza la permanencia en el barrio y } \\
\text { el establecimiento de una mayor calidad de } \\
\text { vida. La proximidad entre las viviendas ha } \\
\text { propiciado el desarrollo de una identidad } \\
\text { espacial que incrementa la reciprocidad e } \\
\text { incluso la transformación del entorno. }\end{array}$ \\
\hline
\end{tabular}




\begin{tabular}{|c|c|c|c|}
\hline Año & Autor & Muestra & Hallazgos \\
\hline 2012 & Cueva & 4 plazas públicas & $\begin{array}{l}\text { Cuatro indicadores de lo simbiótico fue- } \\
\text { ron accesibilidad. movilización, inter- } \\
\text { cambio y apropiación. En el primero, la } \\
\text { peatonización es la estrategia pública } \\
\text { para diluir la segregación e incentivar la } \\
\text { inclusión de los visitantes en los eventos } \\
\text { de las plazas públicas. En el segundo, los } \\
\text { espacios están dotados de mobiliario que } \\
\text { permite la convivencia y el intercambio } \\
\text { de ideas para la apropiación simbólica del } \\
\text { espacio. El transporte colectivo tiene su } \\
\text { base en dichos espacios y ello facilita el } \\
\text { tránsito de pasaje a peatón o a la recrea- } \\
\text { ción. En el tercero, la edificación de igle- } \\
\text { sia, ayuntamiento, bancos, restaurantes y } \\
\text { otros comercios facilitan el intercambio } \\
\text { social. Por último, la apropiación del es- } \\
\text { pacio es el resultado de la accesibilidad, } \\
\text { movilización e intercambio. Las plazas } \\
\text { públicas son centros de reunión, convi- } \\
\text { vencia, comercio, transporte y recreación. }\end{array}$ \\
\hline 2012 & $\begin{array}{l}\text { Urquieta y } \\
\text { Campillo }\end{array}$ & $\begin{array}{l}\text { Mujeres residente de } \\
\text { Monterrey, Nuevo león }\end{array}$ & $\begin{array}{l}\text { Establecieron una relación entre los re- } \\
\text { cursos económicos y la estratificación } \\
\text { social con respecto a la representación de } \\
\text { la ciudad. Las clases bajas percibieron a } \\
\text { la centralidad como un área insegura. Las } \\
\text { clases medias se mostraron preocupadas } \\
\text { por la expansión de la ciudad y sus efec- } \\
\text { tos en el medio ambiente. Respecto a la } \\
\text { expectativas, manifestaron un ideal de } \\
\text { ciudad en la que los espacios permitirían } \\
\text { la convivencia como elemento de inclu- } \\
\text { sión; recuperación de espacios, tranquili- } \\
\text { dad y disfrute. Respecto al derecho a la } \\
\text { ciudad, esta fue representada como un } \\
\text { escenario de libertades en las que son in- } \\
\text { dispensables el acceso al empleo, la edu- } \\
\text { cación y salud universales. }\end{array}$ \\
\hline 2013 & $\begin{array}{l}\text { Vinneta y } \\
\text { maharaj }\end{array}$ & 30 participantes & $\begin{array}{l}\text { La autotracendencia se relacionó positiva } \\
\text { y significativamente con las actitudes ha- } \\
\text { cia sí mismo }(0,73) \text {. }\end{array}$ \\
\hline
\end{tabular}

Fuente: Elaboración propia 